\title{
Fuzzy logic based monitoring system for detecting the concentration of hydrogen cyanide
}

\author{
Nilesh Dashore ${ }^{1}$ and Gopal Upadhyaya ${ }^{2}$ \\ ${ }^{1}$ Dept. of Physics, B.M. Engg. College, Indore-452012; ${ }^{2}$ School of Studies in Physics, Vikram University, Ujjain, India.
}

nk_dashore@yahoo.co.in; gopalujjain@yahoo.co.in

\begin{abstract}
This paper describes a fuzzy logic based monitoring system able to detect, measure the concentration of hydrogen cyanide released into the environment from the exhaust of vehicles, burning nitrogen-containing plastics or from any other source. We applied our suggested model to sensor network. The mobile sensor network is composed of a collection of distributed nodes each of which has limited sensing, intelligence and communication capabilities. An ad hoc wireless network is established among all nodes and each node considers other node as extended sensor.
\end{abstract}

Keywords: Hydrogen cyanide monitoring system; Fuzzy logic control system.

\section{Introduction}

Hydrogen cyanide (with the historical common name of Prussic acid) is a colorless, extremely poisonous liquid that boils at room temperature $\left(26^{\circ} \mathrm{C}\right)$. A hydrogen cyanide concentration of $300 \mathrm{mg} / \mathrm{m}^{3}$ in air will kill a human within about 10 minutes. The toxicity is caused by the cyanide ion, which halts cellular respiration by inhibiting an enzyme in mitochondria called cytochrome c oxidase.

$\mathrm{HCN}$ is used in a variety of manufacturing process viz. pharmaceuticals, methyl methacrylate, sodium cyanide, cyanuric chloride etc. It is being released to environment through insecticide and fungicide applications, cigarette smoke (Fiskel et al., 1981) and metallurgic processes including electro plating (El Ghawabi et al., 1975 and from endogenous content of cassava (Kamalu,1995) or sorghum (Ping Wang et al., 1999). Apart from that, in fires, $\mathrm{HCN}$ is formed in the reaction zone from fuels containing nitrogen $(\mathrm{N})$ and hydrogen $(\mathrm{H})$ atoms. Especially in the burning of wool, nylon, synthetic rubber etc (SP Technical Research Institute of Sweden, 2010). Motor vehicle exhaust emissions are also known to contain HCN (Baum et al., 2007).

\section{Hydrogen cyanide monitoring system}

It consists of the following subsystems:

HCN detector: One of the main detector AreaRAE Steel designed by RAE SYSTEMS San jose is used to measure the concentration of HCN (RAE Systems; www.raesystems.com). The detector AreaRAE Steel consists of an ATEX-certified multi-gas, wireless monitor. Housed in a welded stainless-steel enclosure, it is rugged, portable and weather-resistant, making it suitable for harsh environments. Ideal for hazardous materials and other emergency response monitoring applications, strategically placed AreaRAE Steel monitors can be used to quickly establish a perimeter, allowing first responders to back off to a safe position and monitor readings from up to 3 kilometers away. The photoionization detector (PID) in the AreaRAE Steel can measure parts per million of volatile organic compounds (VOCs). An integrated wireless modem transmits real-time gas measurement data to a base station. The base station can simultaneously control and display readings for up to eight AreaRAE Steels. This provides a multi-threat detection network that can monitor a wide geographic area. Employing mesh networking capabilities, the AreaRAE Steel is designed for extended-range performance in difficult radio environments.

Sensor specifications: The detection range for $\mathrm{HCN}$ :

$0-100 \mathrm{ppm}$ with resolution of $1 \mathrm{ppm}$.

Fuzzy control system: The block diagram of fuzzy control system is as shown earlier (Nilesh \& Gopal, 2009). The sensors input are supplied for fuzzification and then Inference engine is used to find out rule based output and after defuzzification crisp output is controlled to get sensor deployment location (Dashore Pankaj \& Jain Suresh, 2008).

\section{Wireless sensor and ad hoc network}

Wireless sensor networks (WSN) generally consist of a data acquisition network and a data distribution network, monitored and controlled by a management center.

WSN is the most potential technology for very low power ubiquitous networks. Foreseen applications fields include monitoring of remote or hostile geographical regions. WSN may consist of even thousands of small and fully autonomous nodes, which gather sensor information, perform data processing, and communicate with each other (Cui et al., 2004). Sensor networks are the key to gathering the information needed by smart environment. A general setup of a wireless sensor network consists of a large number of sensors randomly and densely deployed in a certain area. Each compact sensor usually is capable of sensing, processing data at a small scale, and communicating through Omni-directional
Research article

CIndian Society for Education and Environment (iSee)
"Fuzzy logic based HCN monitoring system" http://www.indjst.org
Nilesh \& Gopal Indian J.Sci.Technol. 
radio signal. An ad hoc network is formed by wireless mobile nodes (hosts) that operate as terminals as well as routers in the network, without any centralized administration (Lewis et al., 2005). Mobile ad hoc networks provide temporary wireless networking capability in situations where no fixed infrastructure exists. These networks are created spontaneously without any infrastructure. Since an ad hoc network can be deployed rapidly with relatively low cost, it becomes an attractive option for commercial uses such as sensor networks or virtual classrooms.

Wireless ad-hoc sensor networks have recently attracted much interest in the wireless research community as a fundamentally new tool for a wide range of monitoring and data-gathering applications (Mohammad \& Abbas, 2006).

\section{Fuzzy logic control process to detect the concentration of hydrogen cyanide Fuzzyfication}

Fig. 1 -3. Concentration of HCN in air
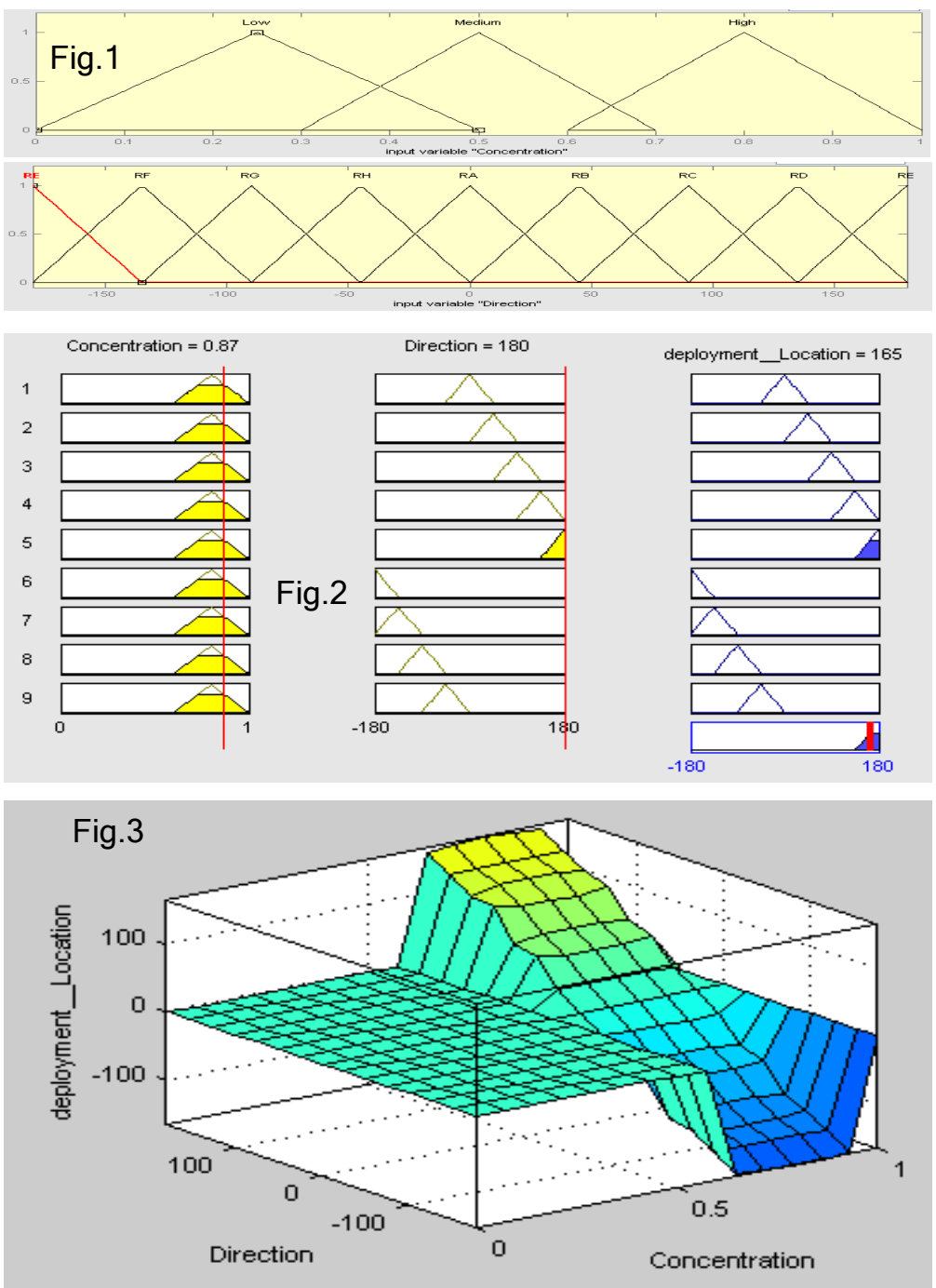

Vol. 3 No. 3 (Mar 2010)

ISSN: 0974- 6846

It comprises the process of transforming crisp values into grades of membership for linguistic terms of fuzzy sets. The membership function is used to associate a grade to each linguistic term. The fuzzification is the first step in fuzzy logic processing involves a domain transformation where the crisp inputs are transformed into fuzzy inputs. To transform crisp inputs into fuzzy inputs, membership function must first be determined for each point.

We define 8 types of linguistic variables as the inputs of fuzzy wireless sensor with related location angle of sensors as RA, RB, RC, RD, RE, RF, RG, RH. The set of sensor values switched at different angles are provides the Input values. The membership function for the location angle set and sensor moving direction set are as shown elsewhere (Nilesh \& Gopal, 2009). The sensor location angles range from $-180^{\circ}$ to $+180^{\circ}$. The membership function of concentration at low, medium and high values between 0 and 1 (Nilesh \& Gopal, 2009).

Fuzzy controller: It evaluates the fuzzy rules with the fuzzy inputs. In this example Fuzzy controller need to convert the input information in to nodes next movement direction. The natural diffusion phenomena of gases and aerosols tend to spread in the environment inducing a concentration gradient that can be used as a clue for tracing emission sources. The rule set should tend to steer all nodes to the vicinity of the highest reported concentration. In our approach we use MATLAB fuzzy logic tool box to generate FLC module. These fuzzy rules are described in IF-THEN form and use the linguistic variables. For an example:

- IF concentration is HIGH and direction is RA THEN move to RA expansion cell.

- IF concentration is $\mathrm{HIGH}$ and direction is RB THEN move to RB expansion cell.

- IF concentration is $\mathrm{HIGH}$ and direction is $\mathrm{RH}$ THEN move to $\mathrm{RH}$ expansion cell.

Defuzzyfication: Since the task of the FLC controller is steering the node's sensor towards the highest reported concentration in our MATLAB flc module, the centre of gravity method is used to get a crisp output to control the next moving direction. The FLC controller uses other nodes current location to find out all expansion cells the node has and uses the moving direction generated by the FLC to define the expansion cell located on the path of the moving direction as the nodes optimal deployment location.

\section{Simulation result}

We applied our suggested model to detect HCN in air and found that our model give satisfactory simulation result (Fig. 1-3). Table1 shows the result for maximum concentration in percentage of 100 ppm.
Research article

CIndian Society for Education and Environment (iSee)
"Fuzzy logic based HCN monitoring system" http://www.indjst.org
Nilesh \& Gopal Indian J.Sci.Technol. 
Table 1. HCN detected in air

\begin{tabular}{|c|c|}
\hline Conc.in \% (of $100 \mathrm{ppm}$ ) & Deployment location \\
\hline 0.81 & $166^{\circ}$ \\
\hline 0.98 & $159^{\circ}$ \\
\hline 0.87 & $165^{\circ}$ \\
\hline
\end{tabular}

\section{Conclusion}

We employed Fuzzy logic system in detection of the concentration of $\mathrm{HCN}$. In this, we present a wireless sensor network to search for higher concentration of $\mathrm{HCN}$. This approach ensures that all nodes of the network maintain a dynamically stable ad hoc wireless communication network for collaborative exploration and information fusion to collect information about the concentration of $\mathrm{HCN}$.

\section{References}

1. Baum MM, John A. Moss, Stephen H. Pastel and Gregory A. Poskrebyshev (2007) Hydrogen cyanide exhaust emissions from in-use motor vehicles. Environ. Sci. Technol. 41 (3), 857-862.

2. Cui $X$, Hardin T, Ragade RK and Elmaghraby AS (2004) A swarm-based fuzzy logic control mobile sensor network for hazardous contaminants localization. In: IEEE Int. Conf. on Mobile Ad Hoc \& Sensor Systems. Oct. 25-27, Univ. of Louisville, KY 40292. pp: 194-203.

3. Dashore Pankaj and Jain Suresh (2009) Fuzzy Rulebased system andmetagraph for risk management in electronic banking activities.IJET Singapore vol1No.2,101-107

4. El Ghawabi SH, Gaafar MA, El-Saharti AA, Ahmed SH, Malash KK and Fares R (1975) Chronic cyanide exposure: a clinical, radioisotope, and laboratory study. Br. J. Ind. Med. 32, 215-219.

5. Fiskel J, Cooper C, and Eschenroeder A (1981) Exposure and risk assessment for cyanide. EPA/440/4-85/008. NTIS PB85-220572.

6. Fungal Genetics \& Biol. 28 (2), 126-134.

7. Kamalu BP (1995) The adverse effects of long-term cassava (Manihot esculenta Crantz) consumption. Int. J. Food Sci. Nutr. 46(1), 65-93.

8. Lewis FL (2005) Wireless Sensor Network. The University of Texas, Arlington.

9. Mohammad AA and Abbas J (2006) "Optimized Forwarding for Wireless Sensor Networks by Fuzzy Inference System" The University of Sydney, NSW, Australia.

10. Nilesh Dashore and Gopal Upadhyay (2009) Fuzzy logic based monitoring system for detecting radon concentration. 2 (5), 29-30.

10.Ping Wang, Robert W. Sandrock and Hans D. VanEtten (1999) Disruption of the cyanide hydratase gene in Gloeocercospora sorghi increases its sensitivity to the phytoanticipin cyanide but does not affect its pathogenicity on the cyanogenic plant
Vol. 3 No. 3 (Mar 2010)

ISSN: 0974- 6846

sorghum. Pradyot Patnaik (2002) Handbook of Inorganic Chemicals. McGraw-Hill, ISBN 0070494398.

12.SP Technical Research Institute of Sweden (2010) Formation of Hydrogen Cyanide in Fires. http://www.sp.se/en/index/research/hydrogen_formatio n/Sidor/default.aspx. Extracted on 20th April 2010.
"Fuzzy logic based HCN monitoring system" http://www.indjst.org
Nilesh \& Gopal Indian J.Sci.Technol. 